\title{
Detection methods of low-speed small objects for panoramic ultrashort pulsed radar
}

\author{
Andrey Ananenkov ${ }^{1, *}$, Pavel Sokolov ${ }^{1}$, Vladimir Nuzhdin ${ }^{1}$, and Dmitry Marin ${ }^{1}$ \\ ${ }^{1}$ Moscow Aviation Institute (NRU), NIO-410, 125993, Moscow, Volokolamskoe sh., h.4, \\ Russian Federation
}

\begin{abstract}
The article discusses ways to improve the detection efficiency of small and low-speed objects in conditions of intense background reflections. The properties of the ultrashort pulse echo signals are investigated. The phenomenon of interfering visibility is investigated.
\end{abstract}

\section{Introduction}

Currently, the problem of ensuring the security of crowded places, territories of various facilities, such as airports, directly related to round-the-clock and all-weather monitoring of low-speed small-sized objects on the background of the earth's surface, is very relevant. Potentially dangerous can be people, vehicles, as well as a new class of threats $[1,2,3]$ small unmanned aerial vehicles (UAVs), which have recently become widespread. Further we will consider the problem of monitoring the airfield.

Airfield monitoring can be carried out using various sensors [4], but the most effective, meeting all-weather requirements and work at any time of the day, are radar tools. One of the most difficult objects from the point of view of detection for radars providing monitoring of the entrusted territory is small UAVs that have the following features: the ability to move at low altitudes at a wide speed range: from fractions to 70-80 meters per second, including the possibility of hanging in place, and small RCS [5]. Due to the small RCS, such objects are extremely difficult to detect in the presence of passive interference from the earth's surface and local objects using traditional radars. Thus, the problem of detecting small-sized low-speed targets in conditions of intense interfering reflections from the underlying surface and local objects with using radars is extremely important.

\section{Ways to improve the detection efficiency of small and low-speed objects}

The solution to the problem of detecting small objects on the background of reflections from the underlying surface by radar means is possible not only by improving the algorithms and devices of the moving target detection (MTD), but also by reducing the level of passive interference at the input of the MTD $\left(P_{c l_{-} i n}\right)$. Reducing of $P_{c l_{-} i n}$ is possible by improving the resolution by the delay, that is, expanding the frequency band of

* Corresponding author: kaf407@mai.ru 
the emitted signal [6]. At least two ways are known to increase the frequency band of the emitted signal: using ultrashort pulse signals (UPS) with high-frequency filling [7] or signals with intrapulse modulation (FCM, FM, LFM). The use of chirp signals requires weight processing to reduce the level of the side lobes of the autocorrelation function $(\mathrm{ACF})$, which leads to a decrease in range resolution and increases the requirements for analog paths, which should provide a low level of frequency and phase distortion. In addition, when using probing signals with intrapulse modulation in the radar, a "blind" zone is formed according to the duration of the signal. UPS with high-frequency filling does not have such drawbacks. UPS will be called a radio signal with RF filling of $\mathrm{mm}$ and $\mathrm{cm}$ range and with a duration of 5-10 ns. It provides high spatial selectivity without the side lobes in the delay selection function.

Interference power at the output of the moving target selector:

$$
P_{c l \_o u t}=P_{c l \_ \text {in }} / K_{s},
$$

where $K_{s}$ is the coefficient of suppression of the echo signal from the underlying surface by the MTD procedure.

The power of passive interference at the input of the MTD system $\left(P_{c l_{-} i n}\right)$ is determined mainly by reflections from the underlying "background" surface $\left(P_{b}\right)$, and is proportional to the spatial resolution of the radar. Reducing the area of the resolution element, implemented in the UPS radar technology, allows to reduce the power of the interference signal from the underlying surface (US) at the input of the radar MTD block. The power of the background component (passive interference) of the reflected echo signal is determined by the following expression:

$$
P_{b}=\frac{P_{p} \cdot G_{a}^{2} \cdot \lambda^{2} \cdot \Delta R \cdot \Delta \theta_{a z} \cdot R \cdot \sigma_{0} \cdot F \cdot K_{S}^{-1} \cdot K_{a c c u m}}{(4 \pi)^{2} \cdot R^{4} \cdot \sin \theta_{e a}}
$$

where: $\Delta R$ - radar resolution by range;

$\Delta \theta_{a z}$ - radar resolution by azimuth;

$\theta_{e a}$ - elevation angle (from the nadir) at which the resolution element is observed;

$F$ - path attenuation;

$P_{p}$ - radar pulse power;

$\lambda-$ is the wavelength;

$\sigma_{0}-$ is the specific RCS (SRCS) of the resolution element;

$G_{a}$ - antenna gain;

$K_{S}$ - is the suppression coefficient of the echo signal from the underlying surface by the MTD procedures;

$K_{\text {accum }}=\sqrt{N_{p}}-$ incoherent accumulation coefficient;

$N_{p} \quad$ - the number of dependent pulses.

The use in the radar of ultrashort probing signals (UPS) with a duration of the order of 5-10 ns, many times shorter than in traditional radars, allows one to reduce the power of passive interference already at the input of the MTD device. 
Along with high resolution in range, the UPS technology has the following specific properties [6]:

- small blind zone (from 5-10 m);

- the possibility of implementing the potential (maximum) resolution for any RCS abrupt transition, which ensures the detection of small targets near objects with significant RCS and against the background of reflections from the underlying surface;

- observation of most objects as multipoint allows us to use radar images to solve problems of recognition and classification of objects types;

- the ability to observe sedentary objects by methods that do not use the Doppler effect;

- high detail of the area radar map and its good coincidence with the topographic map;

- environmental safety;

- good electromagnetic compatibility.

An increase in resolution leads to a decrease in spatial smoothing of the range selection function. This leads to the fact that the radar sections filled with passive interference no longer have a continuous character, i.e., there is a division into areas filled with passive interference and without it. Thus, the phenomenon of interclutter visibility (ICV) occurs. In [8], the ICV phenomenon is mentioned with reference to regions of space not occupied by passive interference. This phenomenon is mentioned in [9] as the distinguishability of passive interference inherent in radars with high spatial resolution. Under the ICV areas one should understand: the zone of shadow and other areas with an average echo intensity at the level of the noise of the receiving and recording equipment. If the located object was not obscured by local objects, the phenomenon of inter-noise visibility can be successfully used in practice to detect objects in the gaps between passive interference. The areas where the level of the reflected signal is at the level of the receiver's own noise can be excluded from processing by the MTD algorithms, which will allow to avoid noise multiplication. For areas filled with passive interference, it is possible to use MTD algorithms that do not use the Doppler effect, such as inter-frame compensation (IFC) [10]. Due to the narrow notch band, such a selector allows to detect both dynamic objects and slowly moving ones, while maintaining a high viewing speed. If you subtract frame from the current one not the previous one, but the received several backwards periods of antenna scanning, it becomes possible to observe newly appearing stationary objects, for example, a dangerous object left behind, an intruder coming out of the corner, rising from the trees and a hovering drone, etc.

\section{Experimental data analysis}

The reflected UPS signals properties study was carried out according to the results of field tests of an experimental sample of the X-band UPS airfield surveillance radar.

Table 1. Main technical characteristics of the UPS airfield surveillance radar.

\begin{tabular}{|l|c|}
\hline Working frequency & $F_{0}=9,8 \mathrm{GHz}$ \\
\hline Pulse duration & $\tau_{\text {imp }}=10 \mathrm{~ns}$ \\
\hline Review Period & $T_{\text {scan }}=1 \mathrm{~s}$ \\
\hline Horizontal beam width & $\theta_{\text {horiz }}=0,7^{\circ}$ \\
\hline Vertical beam width & $\theta_{\text {vert }}=6^{\circ}$ \\
\hline
\end{tabular}


Figures 1 and 2 show the radar and optical images of the located area. Two different probe pulse durations of $10 \mathrm{~ns}$, and $50 \mathrm{~ns}$ was used. It can be seen that an increase in resolution by range leads to a reduction in the proportion of radar elements affected by passive interference.

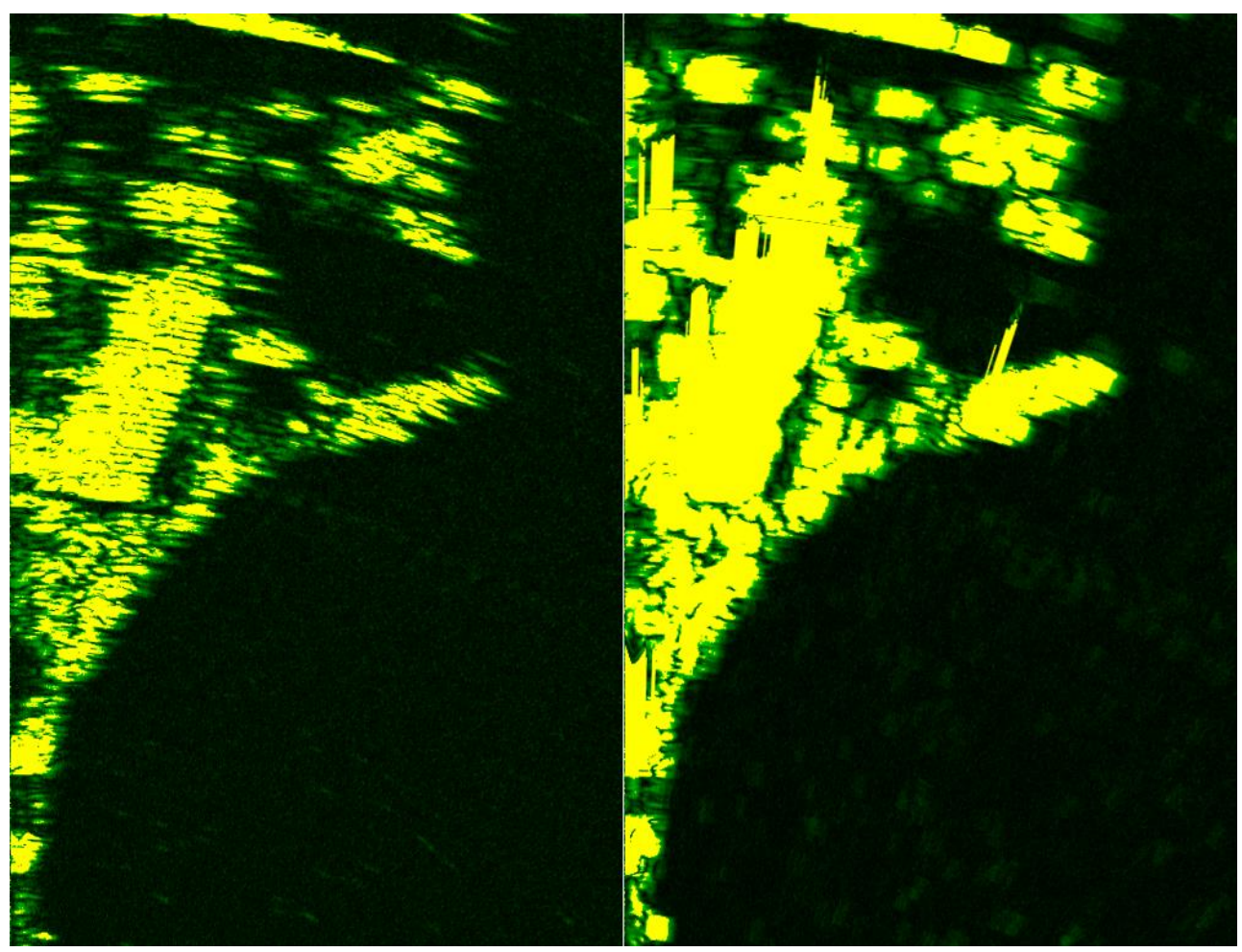

Fig. 1. Radar images of the same located area, obtained with different durations of the probing signal: $10 \mathrm{~ns}$ and $50 \mathrm{~ns}$ (from left to right).

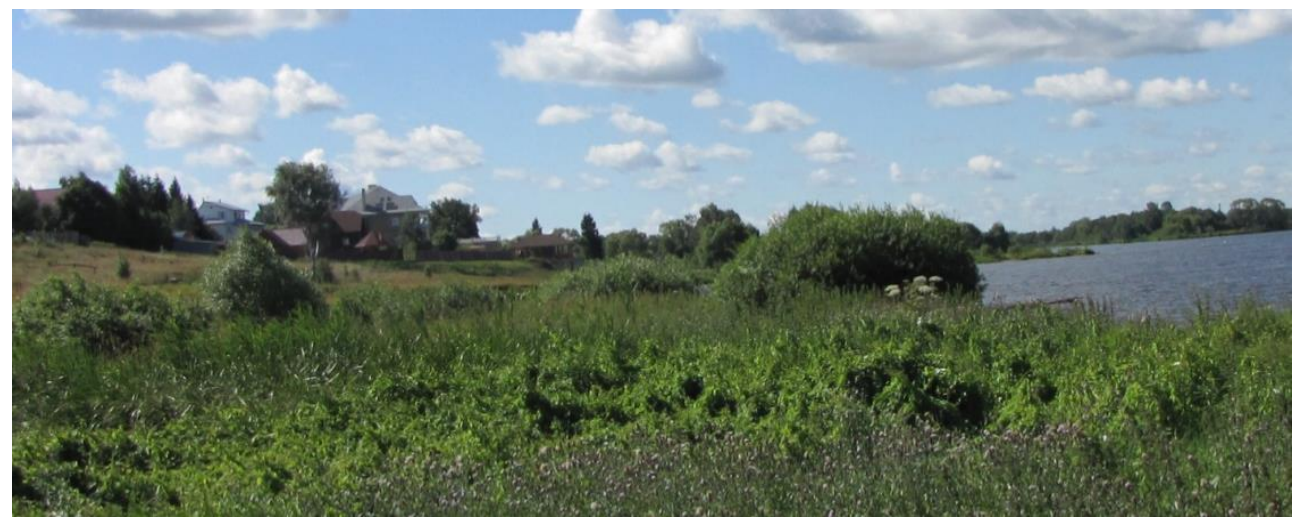

Fig. 2. Optical image of the same location area.

In particular, the exclusion of MTD procedures in those areas of radar data that are not affected by passive interference allows avoiding unnecessary losses in received echo signal processing, which is equivalent to a gain in the radar potential [11]. For these areas, a simple threshold detector can be used. 
An experiment was also conducted to detect small UAVs in conditions of intense reflections of local objects. The tests were carried out in urban areas. Figure 3 shows the radar image, where a person and a UAV are detected against the background of an object with a large RCS (brick wall). Figure 4 shows an optical image of the same scene location.
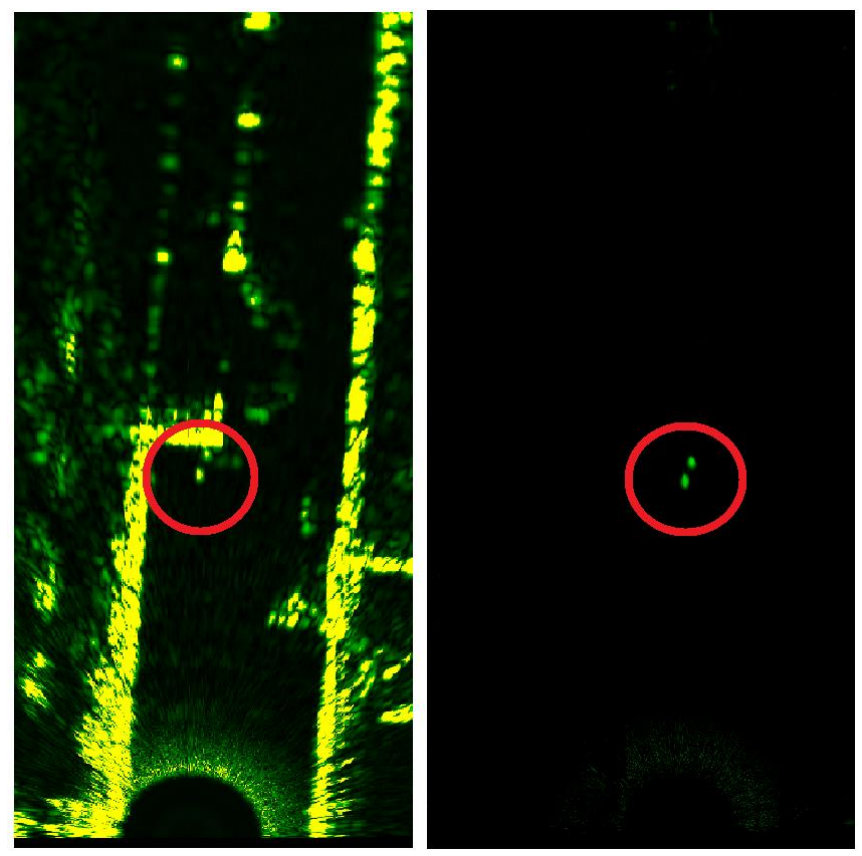

Fig. 3. Locating a small UAV in conditions of intense reflections from surrounding urban objects (buildings). On the left is the primary radar image, on the right is the radar image after application of the MTD procedure

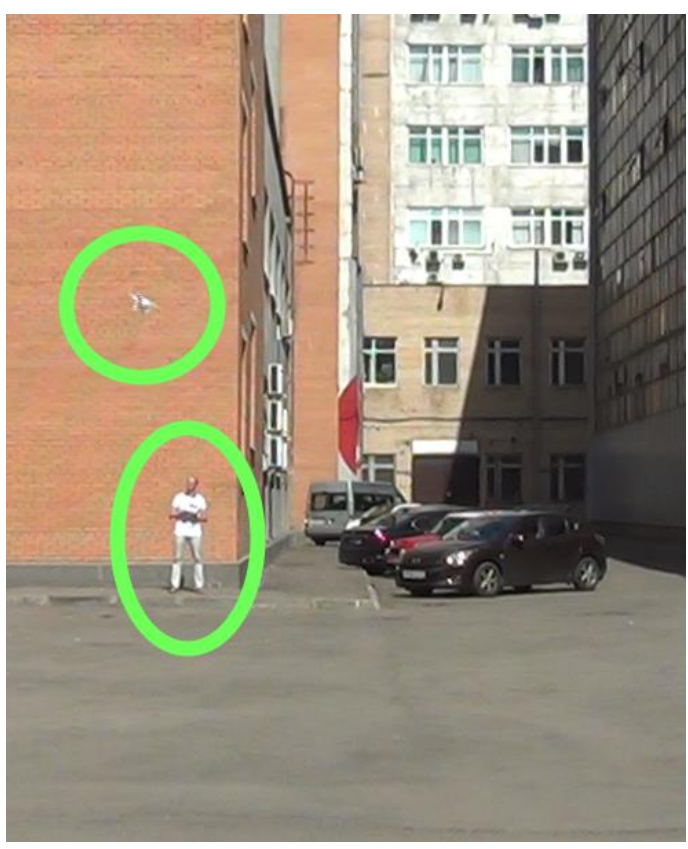

Fig. 4. Optical image of the scene corresponding to a radar image on figure 3. 


\section{Conclusion}

The use of ultrashort pulse probing signal can significantly reduce the level of received background reflections at the input of the secondary processing unit and help to detect small targets against the background of reflections from the underlying surface and local objects.

The use of ultrashort pulse probing signal due to the lack of side lobes of the selection function makes it possible to detect small objects near large ones (with significantly higher RCS).

The algorithm for processing echo signals based on inter-frame subtraction makes it possible to detect both low-speed objects with velocities of fractions of $\mathrm{m} / \mathrm{s}$ and stationary ones (a hovering drone, an object left on the runway) due to subtraction of a frame not taken adjacent but taken a several or more periods ago.

\section{References}

1. M. Coulter, N. Rovnick, How big a threat are drones at airports?, December 20, https://www.ft.com/content/ee9bb576-0455-11e9-99df-6183d3002ee1 (2018)

2. C. Simmons, Drones: protecting airports and aircraft, Aviation Security International. April 15, (2018)

3. Air New Zealand calls for drone legislation after near miss. 27 March (2018) https://www.bbc.com/news/amp/world-asia-43551373 (2018)

4. B. Dietrich, S. Iff, J. Profelt et al., Development of a Local Air Surveillance System for Security Purposes: Design and Core Characteristics, European Journal for Security Research. Vol. 2, Issue 2, pp 71-81, November (2017)

5. M. Krátký, L. Fuxa, Mini UAVs Detection by Radar, International Conference on Military Technologies (ICMT), Brno, May (2015)

6. A.E. Ananenkov, V.M. Nuzhdin, V.V. Rastorguev, V.N. Skosyrev, Highly informative short-range radars MAI Publishing House, Moscow (2018) http://elibrary.mai.ru/ProtectedView/Book/ViewBook/13662 (2018)

7. A.E. Ananenkov, A.V. Konovaltsev, V.M. Nuzhdin, V.N. Skosyrev, Ultrashort pulse radar technology. Status and development trends, Proceedings of the 2nd All-Russian Conference-Seminar, Murom (2006)

8. P.A. Bakulev, Radar systems, M: Radio engineering, p. 62 (2004)

9. M.I. Skolnik, Radar handbook, part 1, p.60, Technosphere, Moscow, (2014)

10. A.E. Ananenkov, D.V. Marin, V.M. Nuzhdin, V.V. Rastorguev, V.N. Skosyrev, Experimental study of reflections from underlying surface in the radar of airfield surveillance, Journal of Radio Electronics, № 2 (2017)

11. V.N. Skosyrev, A.E. Ananenkov, Innovative ways to increase the potential of promising digital radars, Engineering Bulletin, № 12, December (2014) 